\title{
EVALUATION OF HYOID BONE ORIENTATION AFTER ORTHODONTIC CORRECTION OF BIMAXILLARY PROTRUSION
}

\author{
Shereen Mahlawy*
}

\begin{abstract}
Objectives: The aim of this study was to assess the effect of anterior teeth retraction on hyoid bone position in orthodontically treated adults with bimaxillary protrusion.

Materials and Methods: The retrospective data of this study were collected from pre and post treatment lateral cephalograms of twenty Class I bimaxillary protrusive adult female patients with mean age 21.5 years that required extraction of four first premolars and they were treated by preadjusted appliances with maximum anchorage. Lateral cephalograms were traced and analyzed on different linear parameters as (H-SN, H-FH, H-NL, H-Me and H-C3) and angular parameters as (ANB, U1/FH, L1/MP, SNH and MPH). Pretreatment and post treatment parameters variables were statistically analyzed and compared using paired t-test.
\end{abstract}

Results: The mean change of linear measurements (H-SN, H-FH, H-NL, H-C3 and H-Me) pre and post treatment showed statistically significant difference. The mean change of angular measurements ( $\mathrm{SNH}$ and $\mathrm{MPH})$ pre and post treatment showed statistically significant difference and (U1/SN and L1/MP) mean change showed statistically high significant difference while ANB showed non significant difference.

Conclusion: In bimaxillary protrusive adult patients the hyoid bone position is affected by retraction of incisors with extraction of four premolars.

KEYWORDS: Hyoid bone, Cephalometric radiograph, Bimaxillary protrusion

\section{INTRODUCTION}

The hyoid bone has a horse shoe shape that is located at the anterior middle of the neck between the thyroid cartilage and the chin. It is situated at the level of the third cervical vertebra posteriorly and the base of the mandible in the front. ${ }^{1}$ The hyoid bone differs from other facial bones that it is not articulated with any other bone but it is attached to soft tissue via muscles and ligaments. ${ }^{2}$ The hyoid bone position is affected by different muscles as genioglossus, supra and infrahyoid muscles. ${ }^{1,3}$ The head upright posture affects the balance between anterior and posterior muscle tensions of the neck

\footnotetext{
* Lecturer of Orthodontic Department, Faculty of Oral \& Dental Medicine, Fayoum University.
} 
such as masticatory infrahyoid and suprahyoid groups in relation to occipital condyles. ${ }^{4}$ Thus, the position of hyoid bone is an influence of the related tensions of the ligaments, fascia and muscles supported to it. ${ }^{5}$

During sleep, phonation and deglutition, the pharyngeal airway is kept open via the hyoid bone. The position of tongue is maintained by hyoid bone and it also helps during the mandibular movement. Moreover, due to the complex connection between mandible, cervical spine and hyoid bone, it maintains the head posture. ${ }^{6}$

Many studies ${ }^{7,8,9}$ had been done to determine the hyoid bone position at different populations in various malocclusion classes. A study carried upon hyoid bone relationship with base of the skull and mandible reported that the hyoid bone position differed in relation to the anterior base of the cranium. It was found that hyoid bone was placed backward in class II cases, while in class III cases it was placed forward. ${ }^{10}$ Population variations in hyoid bone position exit due to geographical differences. ${ }^{11}$

Lower and upper incisors protrusive proclination, procumbent lips and a disharmonious profile are the main characteristics of bimaxillary protrusion condition. ${ }^{12}$ Such patients who have negative perception of protrusive dentitions and protruding lips often require orthodontic treatment to reduce excessive proclination and improve the profile. ${ }^{13}$

In order to create room for retraction of anterior teeth, four premolars are often extracted, which changes tooth position and also hard and soft tissue components. ${ }^{14}$ The alteration of incisors inclination and soft tissue position could affect potentially the pharyngeal airway and tongue position. ${ }^{15}$

This retrospective study aimed to assess the hyoid bone position upon anterior teeth retraction with four first premolars extraction in bimaxillary protrusion adults.

\section{MATERIALS AND METHODS}

The proposed sample size in this study was 20 which had a power of 80 percent to yield a result which is statistically significant. Sample size estimation based on a previous study. ${ }^{16}$ This comparative retrospective study was ethically approved from Research Ethics Committee of Faculty of Dental Medicine, Al Azhar University Girls Branch and it was carried upon lateral cephalograms pre and post treatment taken from files of previously treated twenty female patients at the outpatient clinic, orthodontic department and they were selected according to the following inclusion criteria:

- Skeletal Class I bimaxillary protrusion

- Extraction of four first premolars

- Age range from 19-24 years

- No or minimal crowding with well aligned arches.

- The dental history was free of previous orthopedic or orthodontic treatment.

- The medical history of patients was free from chronic mouth breathing, tonsillectomy and adenoidectomy.

- Orthodontically treated with preadjusted appliances $(0.022 \times 0.028 \mathrm{inch})$

- Maximum anchorage mechanics.

At natural head position all Lateral cephalograms were taken from the same machine by the same operator. Cephalograms pre and posttreatment were traced and analyzed on different linear parameters as (H-SN, H-NL, H-FH, H-C3 and H-Me) and angular parameters as (ANB, U1/FH, L1/MP, SNH and $\mathrm{MPH})$. Parameters were taken from previous articles. $^{9,} 17$ The Cephalometric landmarks and measurements used for evaluation of hyoid bone 


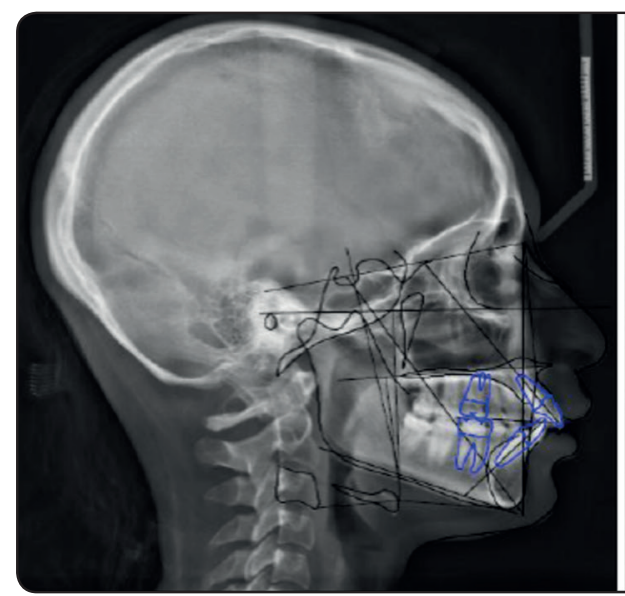

Fig. (1): Cephalometric analysis

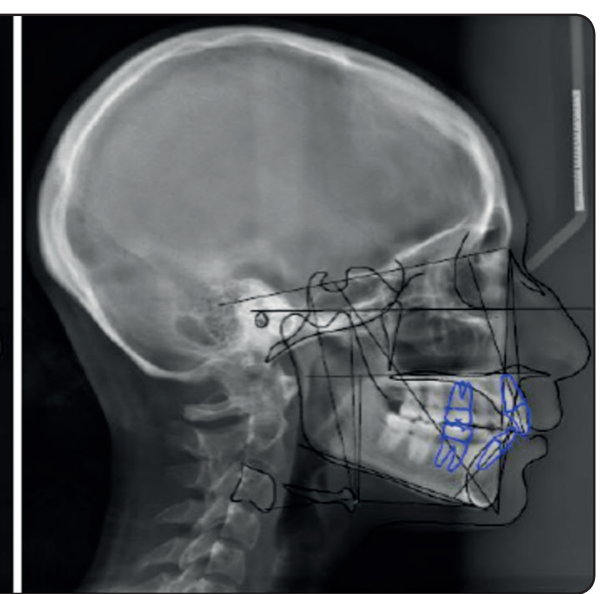

(a) Pre-treatment

(b) Post- treatment position are given in table (1). The cephalograms were digitally traced by a single researcher (Dolphin imaging software). Fig. 1 They were again reevaluated by the same examiner after a week for intraexaminer reliability and the arithmetical means of these readings were taken as the standard value for statistical evaluation and assessment.

TABLE (1): The Cephalometric landmarks and measurements

\begin{tabular}{ll}
\hline H & $\begin{array}{l}\text { (hyoidale) The most anterior superior point on } \\
\text { hyoid bone body. }\end{array}$ \\
\hline C3 & $\begin{array}{l}\text { The most inferior and anterior point on the third } \\
\text { cervical vertebrae. }\end{array}$ \\
\hline SNH & The angle from sella nasion plane to hyoidale. \\
\hline MPH & $\begin{array}{l}\text { The angle from mandibular plane (gonion- } \\
\text { gnathion) to hyoidale. }\end{array}$ \\
\hline H-SN & $\begin{array}{l}\text { A linear perpendicular distance from } \mathrm{H} \text { to the S-N } \\
\text { plane. }\end{array}$ \\
\hline H-NL & $\begin{array}{l}\text { A linear perpendicular distance from H to the nasal } \\
\text { plane. }\end{array}$ \\
\hline H-FH & $\begin{array}{l}\text { A linear perpendicular distance from H to the } \\
\text { Frankfort plane. }\end{array}$ \\
\hline H-C3 & Linear distance between C3 and H. \\
\hline H-Me & Linear distance between the menton and hyoidale. \\
\hline
\end{tabular}

\section{RESULTS}

IBM statistical package for social science (SPSS) was used for data analysis. The values of the arithmetic mean and standard deviation of linear and angular parameters were calculated using descriptive statistics. Paired t test was performed for comparison between different means of angular and linear parameters pretreatment versus posttreatment. The significance level was set at probability less than $5 \%(\mathrm{P} \leq 0.05)$

The means of linear and angular measurements of the study pre and post treatment were tabulated in table (2) and represented in graph (1). The mean change of linear measurements (H-SN, H-NL, H-FH, $\mathrm{H}-\mathrm{Me}$ and $\mathrm{H}-\mathrm{C} 3$ ) showed a statistically significant difference $(\mathrm{p}$ value $=0.019,0.003,0.014,0.003$ and 0.027 respectively). There were a significant increase in means of (H-SN, H-FH, H-N, H-Me), while there was a significant decrease in mean of (H-C3). The mean change of angular measurements (MPH and $\mathrm{SNH}$ ) showed statistically significant difference $(\mathrm{p}$ value $=0.0001$ and 0.001 respectively). The mean change of angular measurements (U1/FH and L1/MP) showed statistically high significant difference ( $p$ value $<0.0001$ ) while (ANB) showed non significant difference. There was a significant increase in mean of (MPH) angle, while there were a significant decrease in means of (SNH, U1/SN and L1/MP) angles. 
TABLE (2): Means of linear and angular measurements pre and posttreatment:

\begin{tabular}{|c|c|c|c|c|c|}
\hline Measurement & Variable & $\begin{array}{r}\text { Pretreatment } \\
\text { Mean } \pm(\text { SD })\end{array}$ & $\begin{array}{r}\text { Posttreatment } \\
\text { Mean } \pm(\text { SD })\end{array}$ & $\begin{array}{c}\text { Mean } \\
\text { change }\end{array}$ & P value \\
\hline \multirow{5}{*}{$\begin{array}{c}\text { Linear } \\
\text { measurements }\end{array}$} & H-SN & $85.30 \pm 2.41$ & $88.50 \pm 5.32$ & 3.2 & $0.019 *$ \\
\hline & H-FH & $72.25 \pm 3.5$ & $74.75 \pm 2.6$ & 2.5 & $0.014 *$ \\
\hline & H-NL & $48.6 \pm 2.1$ & $50.8 \pm 2.3$ & 2.2 & $0.003 *$ \\
\hline & H-C3 & $33.5 \pm 3.5$ & $30.5 \pm 4.7$ & 3 & $0.027 *$ \\
\hline & H-Me & $40.75 \pm 3.7$ & $45.5 \pm 5.6$ & 4.75 & $0.003 *$ \\
\hline \multirow{5}{*}{$\begin{array}{c}\text { Angular } \\
\text { measurements }\end{array}$} & U1/FH & $123.7 \pm 3.42$ & $110.56 \pm 2.45$ & 13.14 & $<0.0001 * * *$ \\
\hline & L1/MP & $103.5 \pm 2.16$ & $97.3 \pm 3.12$ & 6.2 & $<0.0001 * * *$ \\
\hline & ANB & $4.15 \pm 1.25$ & $3.87 \pm 1.52$ & 0.28 & $0.5 \mathrm{NS}$ \\
\hline & SNH & $53 \pm 2.8$ & $49.75 \pm 3.2$ & 3.25 & $0.001 *$ \\
\hline & MPH & $12.75 \pm 2.25$ & $16.75 \pm 3.25$ & 4 & $0.0001 *$ \\
\hline
\end{tabular}

SD: Standard Deviation * $p \leq 0.05$ : Significant

*** P<0.0001: Very high significance NS: non significance

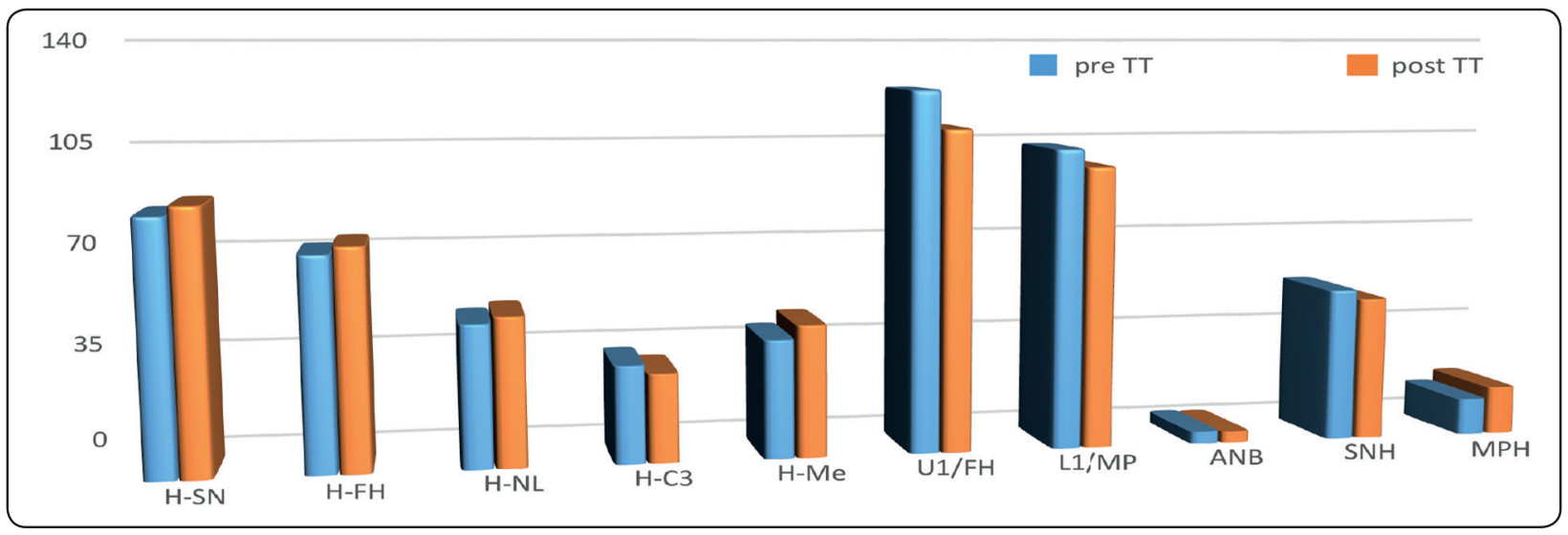

Graph (1): The means of angular and linear measurements pre and post treatment

\section{DISCUSSION}

Various studies $^{7-9}$ have been reported to determine hyoid bone position cephalometriclly in various classes of malocclusions, while this study aimed to investigate the influence of orthodontic retraction of anterior teeth with extraction of four first premolars in bimaxillary protrusion on hyoid bone position which had little literature available.
This study had a retrospective nature and it was held on lateral cephalograms pre and post treatment of patients with Egyptian origin, such that racial and ethnic variation may influence the hyoid bone position. Previous study ${ }^{18}$ on hyoid bone regarding age changes, reported that during the period of growth although mandible and hyoid move away from each other and descend, the hyoid bone relative position does not change. In adult life 
the hyoid bone vertical position is more inferiorly compared to other anatomical structures for both sexes. While, the horizontal position of hyoid bone was stable. Thus in the present study the sample was selected in the adult stage in order to avoid any age changes in hyoid bone orientation related to growth.

In class III patients due to the prognathic mandible the position of the hyoid bone is placed more inferiorly and anteriorly. The muscles of supra hyoid that are attached to the tongue and the mandible placed the hyoid bone more anteriorly according to the theory of functional matrix and thus the position of the bone is changed in relation to Class I patients. While the supra hyoid muscles contracture is lesser in Class II and the hence the hyoid is placed more superiorly and posteriorly. The hyoid bone correlation with various skeletal malocclusion can describe well the functional matrix theory role. ${ }^{9}$

Linear integrations had been used only in a previous study ${ }^{7}$ and the angular measurements had not been used, thus the position of the hyoid bone could not be visualized in all dimensions. While other studies used angular beside linear measurements in hyoid bone orientation..$^{9,17}$ At the present study both the angular and linear measurements were analyzed in order to evaluate the hyoid bone orientation before and after treatment.

A statistically significant decrease was reported at this study in the angle between upper central to the FH plane (U1/ FH) and lower central to mandibular plane (L1/MP). In General most of bimaxillary protrusive patients have good occlusion, thus orthodontic treatment was mainly done for improvement of facial esthetics and correction of the protrusive profile. Post treatment the anterior incisors were in the ideal inclination to satisfy the patient esthetical demands. These findings were in accordance with other studies, ${ }^{16,19}$ which showed a highly significant change in (U1/FH) and (L1/MP) after retraction of anteriors in bimaxillary patients.
In this study the hyoid bone anteroposterior movement was indicated by linear measurements ( $\mathrm{H}-\mathrm{C} 3$ and $\mathrm{H}-\mathrm{Me}$ ) which were analyzed in order to determine the relative horizontal linear change in position of hyoid bone post treatment, the mean change in distance of hyoid bone to cervical vertebrae (H-c3) and to mandible (H-Me) was calculated and statistically analyzed which showed a significant decrease in (H-C3) and a significant increase in $(\mathrm{H}-\mathrm{Me})$ after anterior teeth retraction with four first premolars extraction in adults with bimaxillary protrusion. These results were in agreement with other study which displayed significant decrease in $(\mathrm{C} 3 \mathrm{H})$ where $\mathrm{p}<0.05 .^{19}$

A previous study ${ }^{17}$ reported that the hyoid bone position was located more anteriorly in bimaxillary incisor proclination patients than the group of normal incisor relationship. This shown a highly significant difference.

Whereas vertical movement of hyoid bone which is indicated by linear measurements, The means of (H-SN, H-FH, H-NL) were significantly increased in pre and post treatment which indicates an inferior position of hyoid bone after orthodontic retraction of anterior teeth in bimaxillary protrusion. The hyoid bone downward movement that is reported in this study is in accordance with other studies ${ }^{16,19}$ findings which reported inferior movement of hyoid bone after anterior teeth retraction in bimaxillary protrusion as an adaptation movement which keeps the pharyngeal airway space from tongue encroachment.

The hyoid position is influenced by the related balance between the attached muscle bilaterally from the cranial base and the region of mandibular symphysis. ${ }^{18}$ The retraction of anterior teeth causes the oral cavity anterior boundary to move dorsally and reduces the arch length therefore as an adaptation to this change tongue muscle exert an influence on the hypopharynx and glossopharynx behind them. Before beginning of orthodontic treatment 
and premolars extraction a careful thought of the health of airway is important because the backward movement of teeth in orthodontic treatment can reduce the oral space and also the pharyngeal volume can be restricted. ${ }^{19}$

In the present study the mean of (MPH) angle showed an increase in significance which indicates the inferior movement of hyoid bone post treatment, while there were a significant decrease in means of (SNH) angle which indicates the backward displacement of hyoid bone to its normal position posttreatment and this finding is in accordance with a previous study ${ }^{17}$ which indicates that mobile hyoid bone was anteriorly positioned in bimaxillary incisor proclination individuals than the normal relationship subjects and this may be due to tongue influence on hyoid bone which is displaced anteriorly in bimaxillary incisor proclination. Prospective researches could be done later on using different modalities as cone beam computed tomography and cases follow up for long term could give additional results.

\section{CONCLUSION}

The hyoid bone moves in a downward and backward position upon orthodontic retraction of anterior teeth with extraction of four first premolars in bimaxillary protrusive adults.

\section{REFERENCES}

1. Gray H. Gray's Anatomy of the Human Body, Philadelphia, Lea \& Febiger 1954; 194-195.

2. Romanes GJ. Cunningham's Manual of Practical Anatomy. 14th edition. Vol. 3. New York, NY, USA: Oxford University Press; 1983

3. Yamaoka M, Furusawa K, Uematsu T, Okafuji N, Kayamoto D, Kurihara S. Relationship of the hyoid bone and posterior surface of the tongue in prognathism and micrognathia. J Oral Rehabil. 2003; 30 (9):914-20.

4. Brodie AG: Anatomy and physiology of head and neck musculature. Am J Orthod. 1950; 36: 831-44.

5. Bibby RE, Preston CB. The Hyoid triangle. Am J Orthod. 1981; 80: 92-7.
6. Van L.E, Haxhiu M. A, and Cherniack N. S. Relation between upper airway volume and hyoid muscle length. J Applied Physiology. 1987; 63(4):1443-49.

7. Grant L. E. A radiographic study of hyoid bone position in Angle's Class I, II, and Ill malocclusions, Master's thesis, University of Kansas City, 1959.

8. Adamidis IP, Spyropoulos MN. Hyoid bone position and orientation in Class I and Class III malocclusions. Am J Orhdod Dentofacial Orthop. 1992; 101: 308-12.

9. Kim J, Raval1 N and Patil A .The evaluation of hyoid bone in different skeletal malocclusions and growth patterns in Indian population. Int. J. Adv. Res.2016; 4(9): 876-887.

10. T. Kuroda, E. Nunota, K. Hanada, G. Ito, and Y. Shirataki, "A roentgenocephalometric study on the position of the hyoid bone," Bulletin of Tokyo Medical \& Dental University. 1996; 13:227-43.

11. Rasheed F, Awaisi ZH, Elahi MI, Kanju AH, Sahito ZN, Dayar J: Hyoid Bone Position in Orthodontic Patients. Orthod. J. Nepal.2019; 9(2):20-22.

12. Ma J, Wang L, Zhang W, Chen W, Zhao C, Smales RJ. Comparative evaluation of micro-implant and headgear anchorage used with a pre-adjusted appliance system. Eur J Orthod. 2008; 30:283-87.

13. Kusnoto J, Kusnoto H. The effect of anterior tooth retraction on lip position of orthodontically treated adult Indonesians. Am J Orthod Dentofacial Orthop. 2001; 120:304-7.

14. Sharma JN. Skeletal and soft tissue point A and B changes following orthodontic treatment of Nepalese Class I bimaxillary protrusive patients. Angle Orthod. 2010; 80:9196.

15. Valiathan M, El H, Hans MG, Palomo MJ. Effects of extraction versus non-extraction treatment on oropharyngeal airway volume. Angle Orthod. 2010; 80:1068-1074.

16. Nuvusetty B, Peddu R, Prakash A, Kalyani M.Assessment of changes in pharyngeal airway size and hyoid bone position following orthodontic treatment of Class I bimaxillary dentoalveolar protrusion J Indian Orthod S .2016; (50) 4:215-21

17. Adesina BA, Otuyemi OD, Ogunbanjo BO, Otuyemi DO. Cephalometric assessment of hyoid bone position in Nigerian patients with bimaxillary incisor proclination WACS. 2016; 6 (4):117-135.

18. Durzo CA, Brodie AG. Growth behavior of the hyoid bone. Angle Orthod 1962; 32:193-204.

19. Wang Q, Jia P, Anderson NK, Wang L, Lin J. Changes of pharyngeal airway size and hyoid bone position following orthodontic treatment of Class I bimaxillary protrusion. Angle Orthod 2012; 82:115-21. 